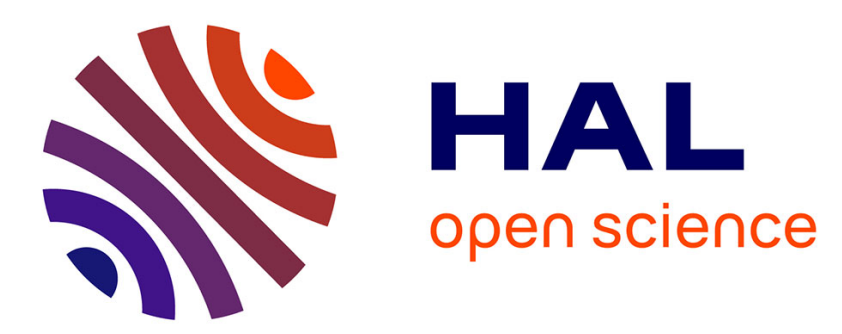

\title{
Propriétés électroniques quasi-bidimensionnelles de couches épitaxiales de GaAs à dopage $\delta$
}

D. Lavielle, J.C. Portal, M. Stohr, S.P. Najda, A. Briggs, G. Gillmann, P. Bois, E. Barbier, B. Vinter

\section{- To cite this version:}

D. Lavielle, J.C. Portal, M. Stohr, S.P. Najda, A. Briggs, et al.. Propriétés électroniques quasibidimensionnelles de couches épitaxiales de GaAs à dopage $\delta$. Revue de Physique Appliquée, 1989, 24 (5), pp.539-543. 10.1051/rphysap:01989002405053900 . jpa-00246079

\section{HAL Id: jpa-00246079 https://hal.science/jpa-00246079}

Submitted on 1 Jan 1989

HAL is a multi-disciplinary open access archive for the deposit and dissemination of scientific research documents, whether they are published or not. The documents may come from teaching and research institutions in France or abroad, or from public or private research centers.
L'archive ouverte pluridisciplinaire HAL, est destinée au dépôt et à la diffusion de documents scientifiques de niveau recherche, publiés ou non, émanant des établissements d'enseignement et de recherche français ou étrangers, des laboratoires publics ou privés. 


\title{
Propriétés électroniques quasi-bidimensionnelles de couches épitaxiales de GaAs à dopage $\delta$
}

\author{
D. Lavielle $\left({ }^{1,2}\right)$, J. C. Portal $\left(\left(^{1,2}\right)\right.$, M. Stöhr $\left({ }^{2}\right)$, S. P. Najda $\left({ }^{2}\right)$, A. Briggs $\left({ }^{2}\right)$, G. Gillmann $\left({ }^{3}\right)$, \\ P. Bois $\left({ }^{3}\right)$, E. Barbier $\left({ }^{3}\right)$ et B. Vinter $\left({ }^{3}\right)$ \\ (1) I.N.S.A.-C.N.R.S., 31077 Toulouse, France \\ (2) S.N.C.I.-C.N.R.S., 38042 Grenoble, France \\ (3) Thomson-CSF, LCR, 91401 Orsay, France
}

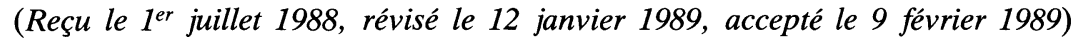

\begin{abstract}
Résumé. - Nous présentons ici une étude des propriétés de transport électronique de structures à dopage planaire Si dans GaAs. Nous utilisons des forts champs magnétiques continus et des basses températures pour caractériser la couche d'accumulation. Nous montrons le caractère bidimensionnel de la conduction et la présence de plusieurs sous-bandes.
\end{abstract}

\begin{abstract}
We report investigations on electronic properties of Si doped GaAs layers. We used high magnetic fields and low temperatures to characterize the accumulation layer. The bidimensional behavior of the conduction and the presence of several electric subbands are evidenced.
\end{abstract}

\section{Introduction.}

Les applications de ces structures sont multiples : super-réseaux « dents de scie » (dopage $\delta$ alternatif $n$ et $\mathrm{p}), \delta$-FET, réalisation de contacts ohmiques non alliés, hétérostructures de haute mobilité $[1,2,3]$. La croissance de ces échantillons, élaborés par épitaxie par jets moléculaires sur GaAs semi-isolant, se présente de la manière suivante :

- croissance d'une couche tampon (1,5 $\mu \mathrm{m})$ d'arséniure de gallium de concentration résiduelle de type $\mathrm{p}$ de l'ordre de $1 \times 10^{15} \mathrm{~cm}^{-3}$;

- arrêt de croissance par fermeture de la cellule de $\mathrm{Ga}$ et dopage par ouverture de celle de $\mathrm{Si}$;

- reprise de la croissance de $\operatorname{GaAs}(0,5 \mu \mathrm{m})$ après fermeture de la cellule $\mathrm{Si}$.

Le flux d'arsenic étant maintenu pendant le dopage $\mathrm{Si}$, les atomes de $\mathrm{Si}$ ont donc tendance à se déposer préférentiellement en site substitutionnel Ga. La cellule d'effusion du Si est portée à $1380^{\circ} \mathrm{C}$ afin de réduire le temps d'interruption de croissance. Le temps de dopage varie de quelques secondes à environ $2 \mathrm{mn}$ dans notre cas. Les très forts dopages obtenus sur quelques plans atomiques induisent une très forte courbure de bande et donnent naissance à un puits de potentiel dans lequel les électrons viennent s'accumuler.
Sur une échelle des temps d'exposition au flux de $\mathrm{Si}$, la concentration électronique mesurée par effet Hall à $300 \mathrm{~K}$ [4] est d'abord proportionnelle au temps de dopage puis sature et décroît enfin au-delà de $90 \mathrm{~s}$. Notre étude porte sur des échantillons dont le dopage en $\mathrm{Si}$ couvre une large gamme ( $\simeq 10^{12} \mathrm{~cm}^{-2}$ jusqu'à quelque $10^{13} \mathrm{~cm}^{-2}$ ). Nous avons étudié les propriétés magnéto-oscillatoires électroniques à $4,2 \mathrm{~K}$ et $1,4 \mathrm{~K}$ puis à très basse température $(\simeq 50 \mathrm{mK})$ à l'aide d'un cryostat à dilution.

\section{Magnétotransport.}

Les spectres de magnétorésistance révèlent la présence de plusieurs séries d'oscillations de périodicités différentes. Ceci démontre la présence de plusieurs sous-bandes électroniques peuplées dans la couche d'accumulation. L'analyse des spectres de Shubnikov-de Haas, du fait de la présence de plusieurs séries d'oscillations (Fig. 1), nécessite l'emploi d'une transformée de Fourier rapide afin de déterminer la population de chaque sous-bande. Cette technique a été discutée par ailleurs par Zrenner et al. [5, 7]. Pour les sous-bandes les moins peuplées, dont le champ fondamental $B_{\mathrm{f}}$, défini ici comme l'inverse de la périodicité en champ magnétique inverse, est 


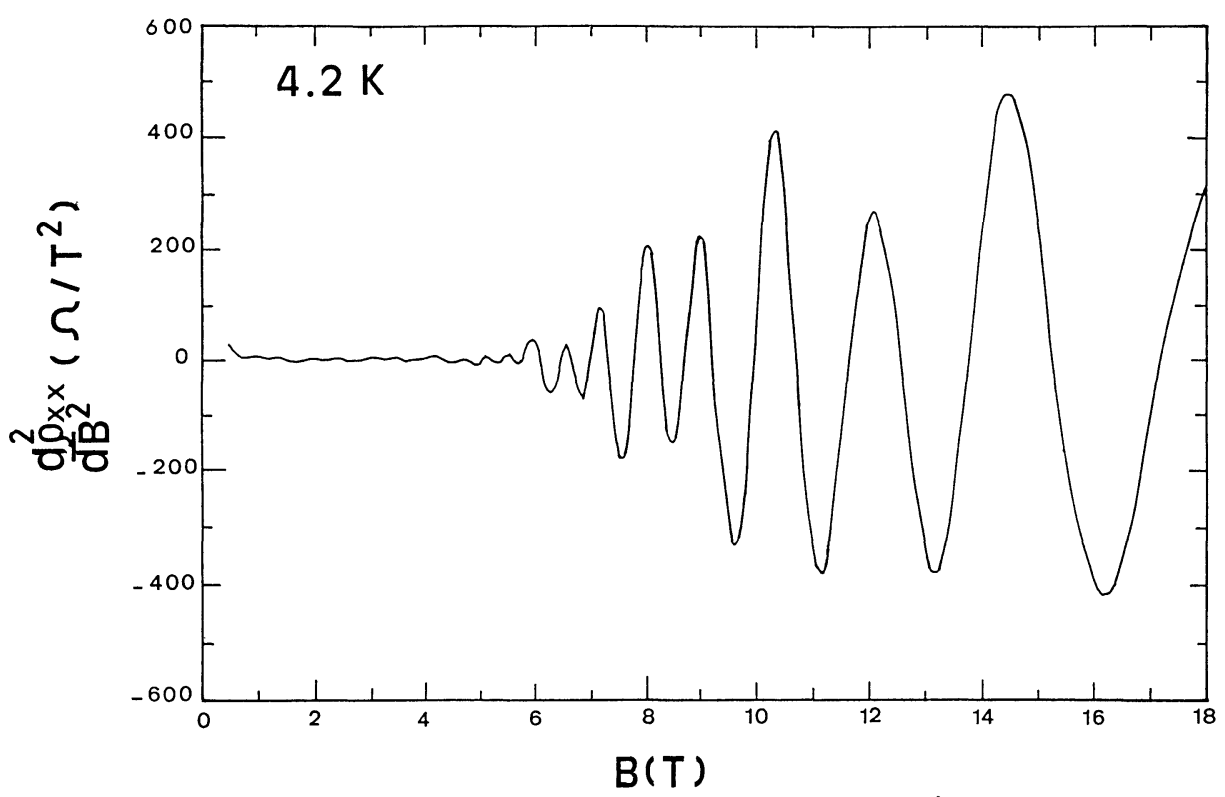

Fig. 1. - Magnétorésistance $\rho_{x x}$ d'un échantillon très dopé présentant plusieurs sous-bandes.

[Magnetoresistance $\rho_{x x}$ from a heavily doped sample with several electric subbands.]

relativement faible $\left(B_{\mathrm{f}} \simeq 2 \mathrm{~T}\right.$ pour $\left.n \simeq 10^{11} \mathrm{~cm}^{-2}\right)$, la condition de quantification $\mu^{*} \mathrm{~B} \gg 1$ nécessaire pour observer les oscillations quantiques peut ne pas être remplie vues les faibles mobilités $\mu \leq 5000 \mathrm{~cm}^{2} /$ V.s. ). Nous considèrerons dans notre cas que les sous-bandes dont la population est inférieure à $8 \times 10^{10} \mathrm{~cm}^{-2}$ ne peuvent être détectées par nos mesures.

Pour les sous-bandes les plus basses, dont la population est très grande, l'utilisation de très forts champs magnétiques est indispensable. En effet les oscillations d'indices de Landau faibles de sousbandes présentant une population supérieure à $5 \times 10^{12} \mathrm{~cm}^{-2}\left(B_{\mathrm{f}} \simeq 100 \mathrm{~T}\right)$ apparaissent à $B>20 \mathrm{~T}$. Le nombre de sous-bandes et les populations respectives des échantillons étudiés sont reportés tableau I.

Sur l'échantillon le moins dopé $(n \simeq$ $7,5 \times 10^{11} \mathrm{~cm}^{-2} ; \mu \simeq 1235 \mathrm{~cm}^{2} /$ V.s. $)$, nous observons un plateau d'effet Hall quantique [6] qui est à $3 \%$ près la valeur théorique du plateau d'indice $i=2$. L'étude de l'effet Hall quantique à très basse température, de l'ordre de $50 \mathrm{mK}$, n'a pas permis de révéler clairement des plateaux d'effet Hall quantique d'indices supérieurs (encart Fig. 2a). Il est remarquable que l'effet Hall quantique soit observé ici dans une situation d'aussi faible mobilité.

L'évolution de la position des minima et maxima des spectres de Shubnikov-de Haas en fonction de l'angle $\theta$ entre le champ magnétique et le plan de dopage a été également étudiée. Le déplacement des oscillations suit bien une loi en $\cos \theta$ (Fig. 3) et confirme le caractère bidimensionnel du gaz. En effet, dans un système bidimensionnel, seule la
Tableau I. - Population respective de chaque sousbande et population de Hall pour des échantillons de dopages différents. $T=4.2 \mathrm{~K}$.

[Population of each subband and Hall density for several samples with various doping levels. $T=4.2 \mathrm{~K}$.]

\begin{tabular}{|c|c|c|cccc|}
\hline Ech. & $\begin{array}{c}\text { Population } \\
\text { de Hall } \\
\left(10^{12} \mathrm{~cm}^{-2}\right)\end{array}$ & $\begin{array}{c}\text { Nombre de } \\
\text { sous-bandes } \\
\text { observées }\end{array}$ & $\begin{array}{c}\text { Population par } \\
\text { effet SDH } \\
\left(10^{12}\right. \\
\left.\mathrm{cm}^{-2}\right) \\
N_{1}\end{array}$ & $N_{2}$ & $N_{3}$ & $N_{\text {tot }}$ \\
\hline 1 & 0,75 & 1 & 0,75 & $*$ & $*$ & 0,75 \\
\hline 2 & 6,37 & 3 & 3,5 & 2 & 0,7 & 6,2 \\
\hline 3 & 18,6 & 3 & 3,7 & 2 & 1 & 6,7 \\
\hline
\end{tabular}

* Les sous-bandes de densité inférieure à $8 \times 10^{10} \mathrm{~cm}^{-2}$ ne peuvent être détectées par nos mesures.

composante perpendiculaire du champ magnétique quantifie les électrons en niveaux de Landau.

Sur cet échantillon faiblement dopé, nous avons de plus mis en évidence un phénomène de photoconductivité persistante à basse température $(4,2 \mathrm{~K})$ que nous attribuons à un processus de séparation spatiale de charges [7]. En effet, après excitation optique d'énergie supérieure à la bande interdite ( $h \nu>1,8 \mathrm{eV})$ des paires électrons-trous sont photogénérées : les électrons sont piégés dans le puits de potentiel et viennent accroître la population bidi- 

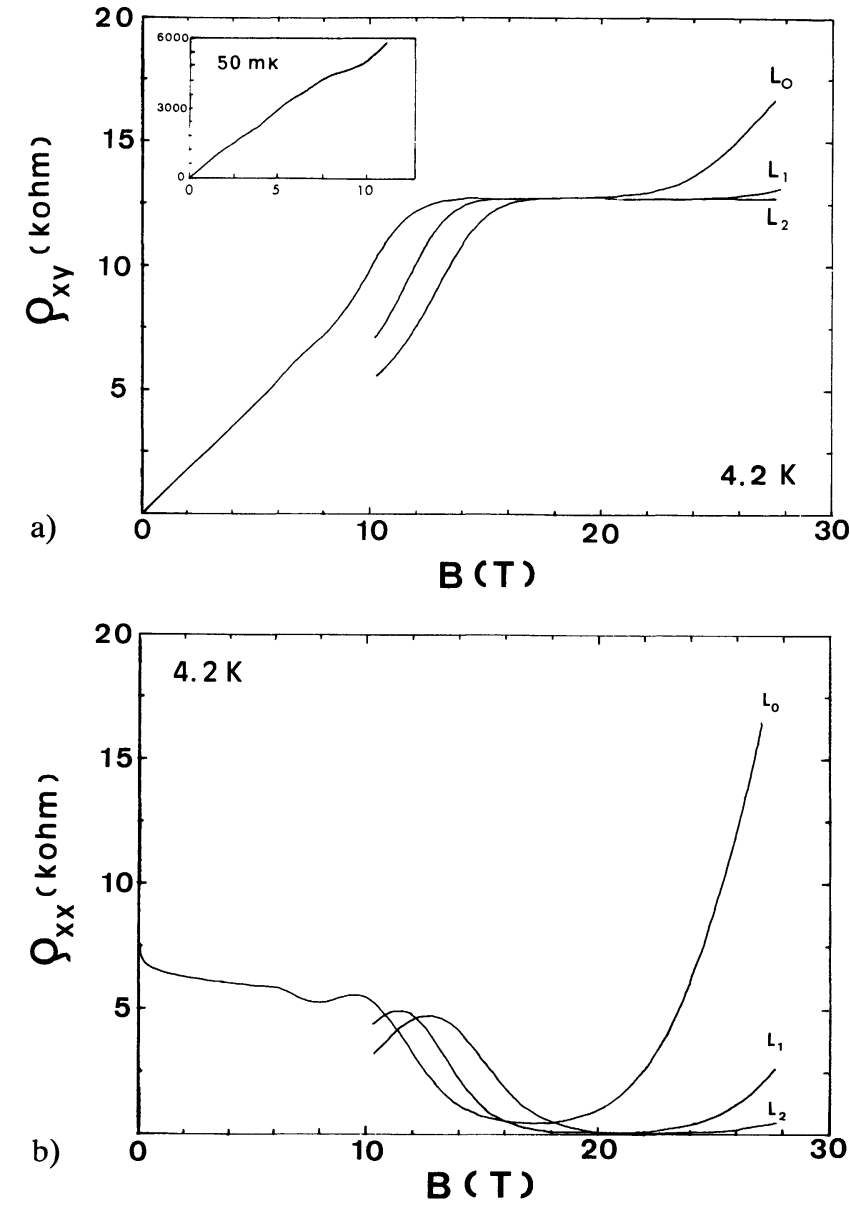

Fig. 2. - Mise en évidence de la photoconductivité persistante : résistivité de Hall $\rho_{x y}$ (a) et magnétorésistance $\rho_{x x}$ (b) en fonction de l'éclairement. $T=4,2 \mathrm{~K}: \mathrm{L} 0$ correspond à l'obscurité ; L1 correspond à un temps d'éclairement $<1 \mathrm{~s} ; \mathrm{L} 2$ correspond à la saturation (temps d'éclairement $>1 \mathrm{~s}$ ).

[Evidence of the persistent photoconductivity effect (PPC). Hall resistivity $\rho_{x y}$ (a) and magnetoresistance $\rho_{x x}$ (b) for different illumination times : L0 : dark; L1 : illumination time $<1 \mathrm{~s} ; \mathrm{L} 2$ : saturated PPC (illumination time $>1 \mathrm{~s})$.]

mensionnelle tandis que les trous sont attirés par la couche tampon de type résiduel $\mathrm{p}$ ou sont piégés à la surface de l'échantillon par des défauts de type accepteurs ionisés. L'augmentation de population est détectée par le déplacement des oscillations de Shubnikov-de Haas et du plateau d'effet Hall quantique vers les forts champs magnétiques en fonction du temps d'éclairement (Figs. 2a, b). La saturation de la photoconductivité permet d'évaluer le nombre d'états de surface accepteurs à un nombre voisin de $4 \times 10^{11} \mathrm{~cm}^{-2}$. Nous observons une augmentation sensible de la mobilité de Hall lorsqu'une sousbande supérieure est peuplée (Tab. II), mettant en évidence les considérations sur la mobilité évoquées ci-dessous.

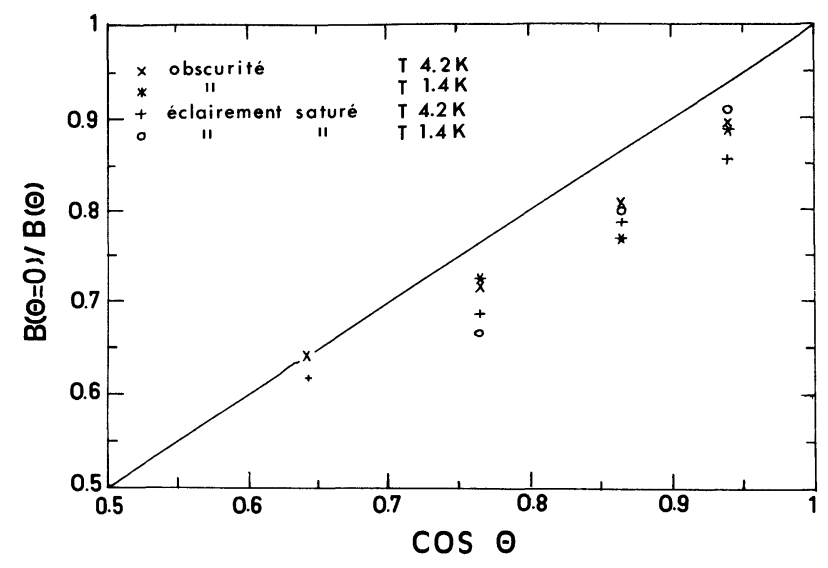

Fig. 3. - Déplacement des oscillations de Shubnikov-de Haas en fonction de l'angle $\theta$ entre le champ magnétique et la normale au plan de dopage.

[Shift from the Shubnikov-de Haas oscillations as a function of the angle $\theta$ between the magnetic field and the surface normal.]

Tableau II. - Population de chaque sous-bande et population et mobilité de Hall pour divers éclairements. L0, L1, L2 correspondent à la figure 2 . L'erreur sur les valeurs des populations est $\pm 0,1 \times 10^{11} \mathrm{~cm}^{-2}$.

[Population of each subband and Hall density and mobility for different optical excitations L0, L1, L2 refer to figure 2 . We estimate the uncertainty on the carrier concentration to be of the order of $\pm 0.1 \times 10^{11} \mathrm{~cm}^{-2}$.]

\begin{tabular}{|c|c|c|c|c|c|}
\hline \multicolumn{3}{|c|}{ Effet Hall } & \multicolumn{3}{c|}{ Effet Shubnikov-de Haas } \\
\hline \multicolumn{3}{|c|}{$\begin{array}{c}\mu_{\mathrm{H}} \\
\left(\mathrm{cm}^{2} / \text { V.s. }\right)\end{array}$} & $n_{\mathrm{H}}$ & $n_{1}$ & \multicolumn{3}{c|}{$\begin{array}{c}n_{2} \\
\left(10^{11} \mathrm{~cm}^{-2}\right)\end{array}$} \\
\hline L0 & 1235 & 7,5 & 7,5 & $*$ & 7,5 \\
\hline L1 & 1385 & 8,2 & 7,3 & 1,1 & 8,4 \\
\hline L2 & 2575 & 11,3 & 8,9 & 3 & 11,9 \\
\hline
\end{tabular}

En effet le gaz électronique étant confiné au voisinage des atomes donneurs qui lui donnent naissance, la mobilité est abaissée par la forte diffusion par les impuretés ionisées. L'extension de la fonction d'onde électronique étant plus petite pour les sous-bandes les plus basses, ce sont celles-ci qui présenteront la mobilité la plus faible du fait du confinement sur les donneurs.

A extension spatiale donnée, un argument de parité permet d'expliquer une différence de mobilité entre sous-bandes. En effet, les fonctions d'onde paires, présentant une probabilité de présence maxi- 
male en $z=0$, entraîneront une mobilité faible, du fait que « statistiquement » les électrons seront confinés plus près des donneurs ionisés. Par contre les fonctions d'onde impaires présentant un nœud de présence en $z=0$ entraîneront une mobilité accrue.

Du fait de la forte différence de mobilité des diverses sous-bandes, l'effet Hall doit être analysé à l'aide d'un modèle multi sous-bande :

$$
n_{\mathrm{H}}=\frac{\sum_{i}\left(n_{i} \mu_{i}\right)^{2}}{\sum_{i} n_{i} \mu_{i}^{2}} \text { et } \mu_{\mathrm{H}}=\frac{\sum_{i} n_{i} \mu_{i}^{2}}{\sum_{i} n_{i} \mu_{i}}
$$

où $n_{i}$ et $\mu_{i}$ sont respectivement la population et la mobilité électronique de chaque sous-bande.

Ceci explique la différence entre la population de Hall et la somme des populations obtenues par effet Shubnikov-de Haas. Cet effet est d'ailleurs clairement mis en évidence dans le tableau I. Dans le noir, une seule sous-bande est peuplée et les populations de Hall et SdH sont égales. Sous éclairement (L1), une deuxième sous-bande est peuplée et la somme des populations de $\mathrm{SdH}$ devient supérieure à celle de Hall. L'augmentation de la mobilité de Hall observée est une preuve expérimentale de la plus forte mobilité de la deuxième sous-bande impaire. Il est à noter également que la variation de mobilité des sous-bandes en fonction de leur état de peuplement peuvent aussi expliquer un accroissement de mobilité. Cependant, pour l'éclairement $\mathrm{L} 1$, la population de la première sous-bande reste égale à sa valeur dans le noir dans la limite de l'incertitude de mesure et la variation de mobilité observée est attribuable uniquement au peuplement de la deuxième sousbande plus mobile. Sa mobilité peut être évaluée à $2050 \mathrm{~cm}^{2} /$ V.s. dans le cadre de l'analyse multi sousbande. Pour l'éclairement L2, la variation de population de la sous-bande fondamentale donc de sa mobilité ne permet plus d'envisager une analyse quantitative des résultats.

Pour les échantillons les plus dopés, la différence entre la population de Hall et la somme des populations de chaque sous-bande mesurées par effet Shubnikov-de Haas laisse à penser que des sousbandes très fortement peuplées ont pu ne pas être décelées et nécessitent l'emploi de champs magnétiques supérieurs.

\section{Etude de la saturation.}

Nous avons par ailleurs utilisé les techniques de pression hydrostatique pour tenter de cerner l'origine de la saturation à fort flux de Si. L'application d'une pression hydrostatique jusqu'à $12 \mathrm{kbar}$ sur des échantillons fortement dopés ou dopés audelà de la saturation n'a pas permis de mettre en évidence une variation de population. Ceci semble devoir écarter l'influence du centre DX dans ce mécanisme de saturation. En effet le centre DX, présent dans GaAs massif fortement dopé, est mis en évidence par le mouvement relatif des vallées $\Gamma$, $\mathrm{L}, \mathrm{X}$ de GaAs sous pression hydrostatique [8]. Le niveau DX, «relié » à $\mathrm{L}$ descend relativement à $\Gamma$ sous pression et vient piéger les électrons de la bande de conduction au-delà d'une certaine pression critique fonction du dopage. Dans nos structures, la connaissance trop faible de la distribution de dopant rend difficile la correspondance 2D-3D. De plus, la saturation peut également être envisagée du point de vue de l'autocompensation ou de la limite de solubilité. Une étude plus complète est donc nécessaire pour mieux cerner le mécanisme de la saturation [9].

\section{Modélisation.}

Devant l'impossibilité expérimentale de connaitre exactement la largeur de la distribution de dopant, Vinter et Zrenner [4, 7] rendent compte de la population des sous-bandes par un modèle selfconsistant dans lequel la distribution de dopant est un paramètre. Nous proposons ici comme alternative une résolution analytique du problème.

Pour une répartition exponentielle du dopant $\left(N_{\mathrm{D}}(x)=N_{\text {DO }} \exp (-|x| / a)\right)$, le potentiel créé par les impuretés ionisées a un profil exponentiel. L'équation de Schrödinger peut être résolue de manière analytique et les solutions sont des fonctions de Bessel. Les énergies propres impaires de l'Hamiltonien sont les zéros des fonctions de Bessel solutions de l'équation de Schrödinger, alors que les zéros des dérivées de ces solutions donnent les énergies propres des états pairs. Ces valeurs tabulées permettent de déterminer la position énergétique de chaque sous-bande électrique. La position du niveau de Fermi est ensuite calculée dans une approche parabolique pour rendre compte de la population électronique totale :

$$
\begin{aligned}
n E_{\mathrm{f}} & =\left(\pi \hbar^{2} / m^{*}\right) \sum_{i} n_{i}+\sum_{i} E_{i} \\
& =\left(\pi \hbar^{2} / m^{*}\right) n_{\mathrm{tot}}+\sum_{i} E_{i}
\end{aligned}
$$

où $n$ est le nombre total de sous-bandes, $E_{i}$ est la position énergétique de la sous-bande $i$.

La population de chaque sous-bande est ensuite calculée séparément :

$$
n_{i}=\left(E_{\mathrm{f}}-E_{i}\right)\left(m^{*} / \pi \hbar^{2}\right) .
$$

Nous avons comparé le modèle analytique et le modèle self-consistant pour un nombre total de donneurs ionisés variant de $2 \times 10^{12}$ à $8 \times 10^{12} \mathrm{~cm}^{-2}$ et une largeur $a$ de la distribution de dopant de $66 \AA$. Le modèle analytique rend compte de manière satisfaisante de la population relative de chaque sous-bande. A titre d'exemple, le tableau III permet 
de comparer les deux modèles pour $7,04 \times 10^{12}$ $\mathrm{cm}^{-2}$ impuretés ionisées.

Tableau III. - Comparaison du modèle analytique et du modèle self-consistant. La largeur de la distribution de dopant est de $66 \AA$. $n_{\text {tot }}=7,04 \times 10^{12} \mathrm{~cm}^{-2}$. $E_{i}$ : position énergétique de la sous-bande $i$ par rapport au niveau de Fermi. $n_{i}$ : population de la sous-bande $i$. $V_{\mathrm{O}}$ : position énergétique de la bande de conduction au centre du puits $(x=0)$ par rapport au niveau de Fermi.

[Comparison between the analytical and the selfconsistent model. The spread-out of the donor sheet is $66 \AA$. $n_{\text {tot }}=7.04 \times 10^{12} \mathrm{~cm}^{-2} . E_{i}$ : energetic position from the subband $i$ (Energy reference : Fermi level). $n_{i}$ : population from the subband $i . V_{\mathrm{O}}$ : energetic position from the conduction band at the bottom of the well $(x=0)$ (Energy reference : Fermi level).]

\begin{tabular}{|c|c|c|c|c|c|c|c|c|}
\hline Modèle & $E_{\mathrm{f}}$ & $V_{\mathrm{o}}$ & $\begin{array}{c}E_{\mathrm{O}} \\
(\mathrm{meV})\end{array}$ & $E_{1}$ & $E_{2}$ & $\begin{array}{l}n_{\mathrm{O}} \\
\quad(10\end{array}$ & $\begin{array}{c}n_{1} \\
12 \mathrm{~cm}\end{array}$ & $\begin{array}{c}n_{2} \\
\left.\mathrm{n}^{-2}\right)\end{array}$ \\
\hline Analytique & 0 & $-261,1$ & $-167,1$ & $-62,6$ & -22 & 4,67 & 1,75 & 0,62 \\
\hline Self-cons. & 0 & -260 & -168 & -65 & -25 & 4,52 & 1,80 & 0,72 \\
\hline
\end{tabular}

\section{Conclusion.}

Nous avons présenté une étude de magnétotransport sur des structures à dopage « $\delta$ » Si dans GaAs. Nous avons mis en évidence un plateau d'effet Hall quantique et un effet de photoconductivité persistante sur l'échantillon le moins dopé. Nos techniques nous ont permis par ailleurs de caractériser plusieurs échantillons présentant un dopage important de l'ordre de $10^{13} \mathrm{~cm}^{-2}$. Enfin un modèle analytique est présenté. Il est comparé au modèle self-consistant et rend compte de manière satisfaisante de la position relative de chaque sous-bande.

\section{Remerciements.}

Les auteurs remercient J. C. Vallier, M. Ohl, J. C. Picoche pour leur assistance technique lors des expériences sur la bobine hybride du Service National des Champs Intenses à Grenoble.

Les auteurs tiennent à remercier le Conseil Régional de Midi-Pyrénées et la Commission de la Communauté Européenne pour leurs soutiens financiers. S. P. Najda remercie la Royal Society pour son aide financière. D. LAVIELLE tient à remercier Thomson-CSF pour son soutien financier.

\section{Bibliographie}

[1] Schubert E. F. et Ploog K., Jpn. J. Appl. Ph. 24 (1985) L 608.

[2] Ploog K., J. Cristal Growth 81 (1987) 304.

[3] Schubert E. F. et al., Appl. Phys. Lett. 49 (1986) 292.

[4] Gillmann G. et al., Appl. Phys. Lett. 52 (1988) 12.

[5] Zrenner A. et al., ICPS San Francisco (1984).
[6] Gillmann G. et al., Semi Sci. Techn. 3 (1988) 620622.

[7] Zrenner A., Thèse Université technique de Münich (1987).

[8] Maude D. K. et al., Phys. Rev. Lett. 59 (1987) 7.

[9] Zrenner A. et al., GaAs and Rel. Comp., Heraklion Crete (1987). 